University of Nebraska - Lincoln

DigitalCommons@University of Nebraska - Lincoln

$4-1-2000$

\title{
DENSE AND RELATIVISTIC PLASMAS PRODUCED BY COMPACT HIGH-INTENSITY LASERS
}

Donald Umstadter

University of Nebraska-Lincoln, donald.umstadter@unl.edu

Shouyuan Chen

University of Michigan-Ann Arbor, schen6@unl.edu

G. Ma

Center for Ultrafast Optical Science, University of Michigan, Ann Arbor, MI

Anatoly Maksimchuk

University of Michigan, tolya@umich.edu

G. Mourou

Center for Ultrafast Optical Science, University of Michigan, Ann Arbor, MI

See next page for additional authors

Follow this and additional works at: https://digitalcommons.unl.edu/physicsumstadter

Part of the Physics Commons

Umstadter, Donald; Chen, Shouyuan; Ma, G.; Maksimchuk, Anatoly; Mourou, G.; Nantel, M.; Pikuz, S.; Sarkisov, G.; and Wagner, R., "DENSE AND RELATIVISTIC PLASMAS PRODUCED BY COMPACT HIGHINTENSITY LASERS" (2000). Donald Umstadter Publications. 55.

https://digitalcommons.unl.edu/physicsumstadter/55

This Article is brought to you for free and open access by the Research Papers in Physics and Astronomy at DigitalCommons@University of Nebraska - Lincoln. It has been accepted for inclusion in Donald Umstadter Publications by an authorized administrator of DigitalCommons@University of Nebraska - Lincoln. 


\section{Authors}

Donald Umstadter, Shouyuan Chen, G. Ma, Anatoly Maksimchuk, G. Mourou, M. Nantel, S. Pikuz, G.

Sarkisov, and R. Wagner 
The Astrophysical Journal SUPPLEMENT Series, 127:513-518, 2000 April

(c) 2000. The American Astronomical Society. All rights reserved. Printed in U.S.A.

\title{
DENSE AND RELATIVISTIC PLASMAS PRODUCED BY COMPACT HIGH-INTENSITY LASERS
}

\author{
D. Umstadter, S.-Y. Chen, G. Ma, A. Maksimchuk, G. Mourou, M. Nantel, S. Pikuz, G. Sarkisov, and R. Wagner \\ Center for Ultrafast Optical Science, University of Michigan, Ann Arbor, MI 48109-2099; dpu@umich.edu \\ Received 1999 January 19; accepted 1999 November 24
}

\begin{abstract}
High-intensity lasers interacting with plasmas are used to study processes in the laboratory that would otherwise only occur in astrophysics. These include relativistic plasmas, electron acceleration in ultrahigh field-gradient wake fields, pressure ionization and continuum lowering in strongly coupled plasmas, and X-ray line emission via Raman scattering.

Subject headings: acceleration of particles - MHD — plasmas — relativity
\end{abstract}

\section{INTRODUCTION}

Many of the phenomena that occur in the extreme conditions of high-intensity laser-plasma interactions would otherwise only occur in the astrophysical realm. In this paper, we discuss several examples that can be classified into two categories: relativistic plasmas and dense plasmas.

\section{RELATIVISTIC PLASMAS}

In the focus of an ultrashort-pulse high-power laser, electrons oscillate at close to the speed of light, giving rise to novel effects in a new regime of laser intensity $\left(I>10^{18} \mathrm{~W}\right.$ $\mathrm{cm}^{-2}$; Umstadter \& Norris 1997). For instance, above the relativistic self-focusing threshold (a few terawatts), a laser is observed to self-guide over many Rayleigh ranges, boring a hole in the plasma in which it propagates, driving a laser wake field, and generating a well-collimated beam of relativistic electrons (Umstadter et al. 1996). The electrons are accelerated from rest to $\mathrm{MeV}$ energies in a micron distance, corresponding to accelerations of $10^{21} \mathrm{~g}$. In laser/wake field-generated plasma waves, electrostatic field gradients are measured to be $E \geq 200 \mathrm{GV} \mathrm{m}^{-1}$, the highest terrestrial fields ever recorded. Due to the enormous pressure (Gbar) exerted radially by the self-focused laser pulse on the plasma, both electrons and ions are expelled from the path of the laser (Chen et al. 1998). The MeV-energy ion "shock" front then interacts with the surrounding plasma. Similar phenomena might be also be produced when strong electromagnetic bursts propagate through the interstellar medium.

Recently, we have shown that an accelerated electron beam appeared to be naturally collimated with a lowdivergence angle (less than $10^{\circ}$ ) and that it had over $1 \mathrm{nC}$ of charge per bunch. Moreover, acceleration occurred in this experiment only when the laser power exceeded a certain critical value $P_{c}$, the threshold for relativistic self-focusing. In what follows, we discuss in detail some of these experimental results.

In these experiments, we used a $\mathrm{Ti}$ :sapphire/ $\mathrm{Nd}$ :glass laser system based on chirped-pulse amplification that produces $3 \mathrm{~J}, 400 \mathrm{fs}$ pulses at $1.053 \mu \mathrm{m}$. The $43 \mathrm{~mm}$ diameter beam was focused with an $\mathrm{f} / 4$ off-axis parabolic mirror to $r_{0}=8.5 \mu \mathrm{m}\left(1 / e^{2}\right)$, corresponding to vacuum intensities exceeding $4 \times 10^{18} \mathrm{~W} \mathrm{~cm}^{-2}$. This pulse was focused onto a supersonic helium gas jet with a sharp gradient $(250 \mu \mathrm{m})$ and a long, flat-topped interaction region $(750 \mu \mathrm{m})$. The maximum density varies linearly with a backing pressure that is up to the maximum backing pressure of 1000 pounds per square inch, and an underdense plasma at $3.6 \times 10^{19}$ $\mathrm{cm}^{-3}$ is formed by the foot of the laser pulse that is tunnelionizing the gas. This plasma density corresponds to a critical power of $P_{c}=470 \mathrm{GW}$. A sharp gradient and long interaction region are found to be essential.

The electron-beam profile was measured using a scintillating screen (LANEX) imaged by a CCD camera (Wagner et al. 1997). The LANEX is placed behind an aluminum sheet that blocks the laser light, so only electrons greater than $100 \mathrm{keV}$ can be imaged. Analysis of the electron spectrum indicates that the bulk of the electrons that create an image on the screen are in the $100 \mathrm{keV}-3 \mathrm{MeV}$ range. We have found, using aluminum absorbers, that the electron divergence does not depend on electron energy in this range. At low power $\left(<5 P_{c}\right)$, the electron beam has a Gaussian-like profile with a $10^{\circ}$ radius at half-maximum (see Fig. 1). As the laser power increases and the plasma channel length increases to $\sim 250 \mu \mathrm{m}$, a second peak seems to grow out of the low-power profile. Ultimately, at the highest laser powers and longest channel lengths, the divergence decreases to $5^{\circ}$, and the profile becomes more Lorentzian-like. The electron-beam divergence should decrease as the longitudinal energy of the electrons increases since the space charge will be less and the relative transverse momentum decreases because of the longer accelerating length. However, there should be a minimum divergence due to the space charge effect after the electrons leave the plasma. This effect is significant since the electrons are in the few $\mathrm{MeV}$ range (small $\gamma$ ) and the peak current is high (large number of electrons in a short bunch). We have roughly estimated the space charge divergence to be $6^{\circ}$ by assuming $10^{9}$ electrons at $1 \mathrm{MeV}$ in a $1 \mathrm{ps}$ bunch (note that $\theta_{\text {hwhm }} \propto\left[N / \tau_{e}(\beta \gamma)^{3}\right]^{1 / 2}$, where $N$ is the number of electrons, $\tau_{e}$ is the electron-bunch duration, and $\beta \gamma$ is the normalized momentum of the electrons). The electron-beam emittance can be found from the measured divergence angle and the radius of the plasma channel, and in the best case $\left(5^{\circ}\right.$ halfangle and $5 \mu \mathrm{m}$ half-maximum radius), the calculated emittance $\left(\epsilon=r_{0} \theta_{\mathrm{hwhm}}\right)$ is $0.4 \pi \mathrm{mm} \mathrm{mrad}$.

In ponderomotive self-channeling, the laser ponderomotive force expels electrons from the axis (the ions do not move much because of their greater mass) and prevents their return, despite the Coulomb force, which arises from charge separation. If the laser-pulse duration is long enough, the ions can start to move out as a result of this Coulomb force and gain momenta during the process. After the laser pulse is gone, electrons quickly return in order to neutralize the bare ions. However, the ions (and electrons) keep moving out of the axis as a result of the ion momen- 


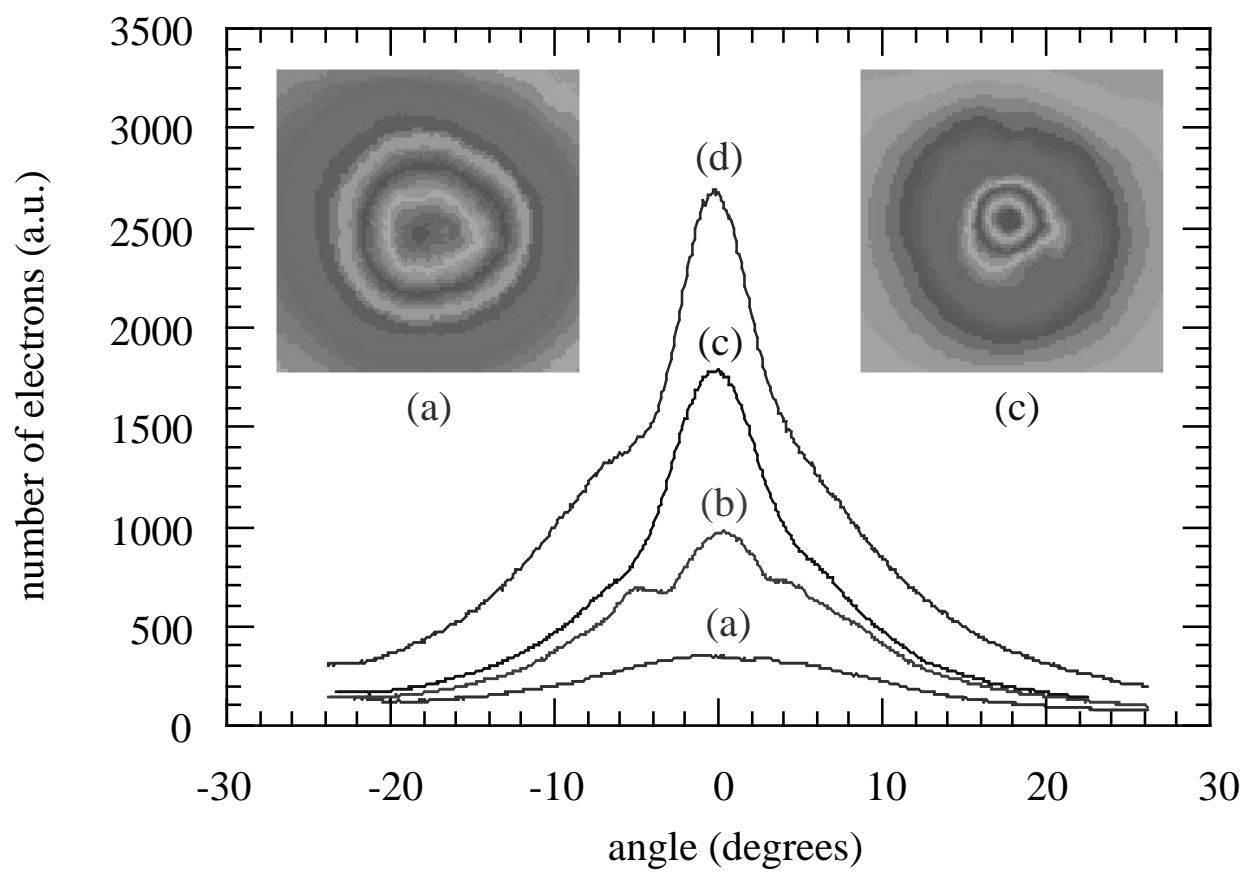

Fig. 1. - Electron-beam divergence as a function of laser power. The various curves represent laser powers of $(a) P / P_{c}=3.4,(b) 5.0,(c) 6.0$, and $(d) 7.5$. The two insert figures show the complete beam images for curves $a$ and $c$.

tum gained during the laser pulse. This is predicted to lead to the formation of a plasma-density depression on-axis (Barnes, Kurki-Suonio, \& Tajima 1987; Pukhov \& Meyerter-Vehn 1996), which becomes deeper and wider with time.

In order to observe the formation and evolution of the density-depression and ion blowout, probing interferometry and shadowgraphy were used. Two-dimensional images of the plasma-density distribution were obtained in this way at different times. A probe pulse (400 fs, $1.053 \mu \mathrm{m}$ ) is obtained by splitting $5 \%$ of the pump pulse, sending it into a delay line and crossing it perpendicularly with the pump pulse in the interaction region. A lens was used to image the probe pulse in the plasma region to a CCD camera, forming shadowgrams. Interferograms were obtained by use of two glass wedges forming a vacuum wedge gap after the lens. To get quantitative measurements of the evolution of the plasma waveguide, a two-dimensional plasma-density distribution was obtained by means of a fringe-tracking program (to get a two-dimensional phase-shift distribution) in conjunction with an algorithm used to perform the Abel integration. Figure 2 shows the two-dimensional plasma-density distribution (cylindrically symmetric) evolving in time. As can be seen, the density depression on-axis becomes deeper and the width of the channel becomes larger as time goes by. At about a 40 ps delay, a plasma waveguide of $800 \mu \mathrm{m}$ in length is formed, which has an on-axis plasma density less than $10^{18} \mathrm{~cm}^{-3}$ and a channel width of $30 \mu \mathrm{m}$. In Figure 2, the waveguide length is equal to the self-channeling length of the pump pulse.

At $2.5 \mathrm{TW}$ laser power, of which $45 \%$ is guided in the channels, the mean ion velocity is about $1 \mu \mathrm{m} \mathrm{ps}^{-1}$. This is consistent with the measured speed of waveguide formation $\left(\sim 1 \mu \mathrm{m} \mathrm{ps}^{-1}\right)$. Figure 2 also shows the increase of the diameter of the plasma region with time at a speed of approximately $1 \mu \mathrm{m} \mathrm{ps}^{-1}$ (about 10 times the thermal expansion velocity at a temperature of $100 \mathrm{eV}$ ). This expansion is due to the production of new plasma in the radial direction caused by collisional ionization. The size of the initial plasma region is determined by ionization by the unguided part (55\% at high powers) of the laser beam, which is defocused to about $100 \mu \mathrm{m}$ FWHM. This gives a laser intensity of $1.5 \times 10^{16} \mathrm{~W} \mathrm{~cm}^{-2}$, which is enough to ionize the entire $100 \mu \mathrm{m}$ diameter plasma region.

The accelerated ions that were produced in the experiments on relativistic plasmas interact with the surrounding gas and plasma to promote bound electrons to excited states. When the electrons eventually radiatively decay to the ground state, they emit photons, creating a glow that can be observed with a CCD camera. We are currently making such measurements in order to test some of the theories on the shock kinetics in neutral and ionized gases. This may help astrophysicists understand how shocks from supernova explosions interact with ejecta and the interstellar medium.

As another example, Raman scattering by the strong electromagnetic fields of intense laser beams has been shown to cause the emission of X-ray satellites that are normally forbidden by dipole selection rules. The observations of these satellites in X-ray astronomical spectra might give clues about the presence of strong fields in the vicinity of the emitting source. The satellites that are induced by lasers can be used to measure the energies of metastable states of highly stripped ions with high accuracy. They might also be observed in spectra of light emitted from astrophysical bodies in which strong electric fields perturb the ions, and thus they form a basis for the measurement of such fields.

\section{DENSE PLASMAS}

Detailed studies of strongly coupled plasmas are now being made with table-top lasers at university laboratories, permitting tests of previously unverified theoretical predictions about pressure ionization and continuum lowering. Such plasmas typically occur in dwarf stars and Jovian planets. Compact laser systems have also been used to show 

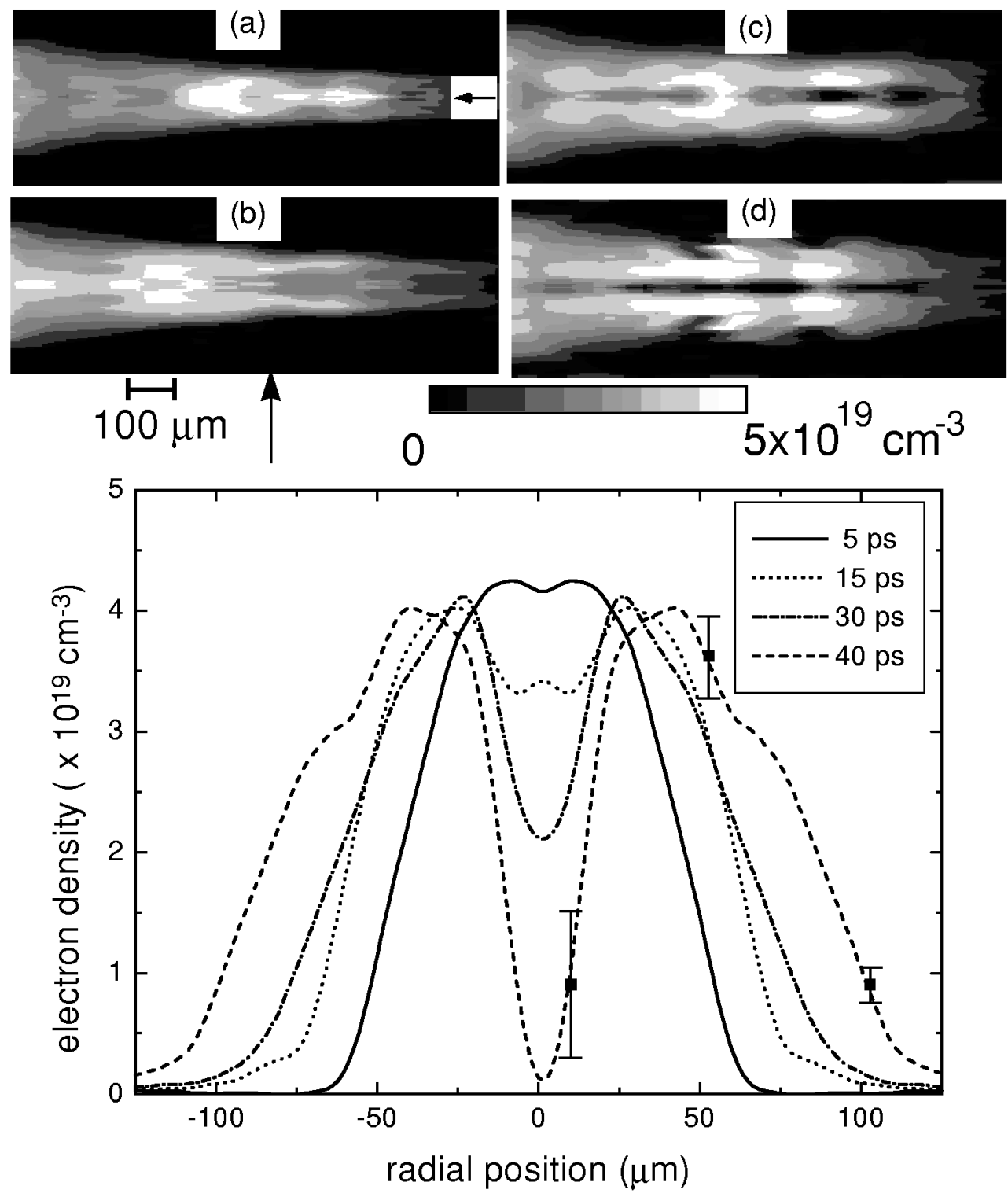

FIG. 2.-Two-dimensional plasma-density distribution for $2.5 \mathrm{TW}$ laser power and $2 \times 10^{19} \mathrm{~cm}^{-3}$ gas density at different times: (a) 5 , (b) 15 , (c) 30 , and (d) $40 \mathrm{ps}$. Line-outs of the cross sections at the position indicated by the vertical arrow are shown along with the error bars at three selected positions.

how X-ray opacities are affected by the pressure changes of ionized gases; specifically, the position of the absorption edge is seen to shift. Such measurements are critical to spectroscopic measurements in astrophysics, which are used to estimate the velocities of, and distances to, stars and other luminous objects.

Strongly coupled plasmas (SCPs) are found in stellar and Jovian planet interiors (Ichimaru 1982), inertial confinement fusion (ICF) pellets, and are essential for a thorough understanding of compressed material equations of state. These plasmas are characterized by high densities and/or relatively low temperatures, such that the Coulomb potential energy between the particles exceeds their kinetic energy, and thus the coupling parameter $\Gamma=\left(Z^{2} e^{2}\right) /\left(r_{i} k T\right)$ (where $r_{i}$ is the ion-sphere radius) is larger than one. Unfortunately, relatively little experimental work on strongly coupled plasmas (DaSilva et al. 1989; Leboucher-Dalimier et al. 1994; Workman et al. 1997) has been done up to now, as these studies are rendered difficult by the need to obtain and diagnose high-density/low-temperature conditions simultaneously. This is particularly true for subpicosecond laser-produced plasmas, which are increasingly important in applications such as fast-ignitor ICF, ultrafast X-ray sources, and ion acceleration. Pressure ionization and line merging are two spectroscopic effects arising from the deformation of the ionic potential by the plasma fields in SCPs; although both have been used for density diagnostics, independent plasma diagnostics must be used to ensure their validity in the strongly coupled regime.

We recently reported X-ray and ultraviolet (XUV) spectra emitted from a strongly coupled laser-produced plasma, making use of two novel experimental techniques: (1) a high-contrast, high-seed injection short-pulse laser to achieve nearly solid density and (2) a jitter-free X-ray streak camera to achieve both a high signal-to-noise ratio and an ultrahigh temporal resolution (in picoseconds). This combination, short laser pulses and high temporally and spatially resolved spectroscopy, makes it possible to study the 
dynamics of SCPs over a wide range of densities and temperatures, which makes for an interesting test bed for atomic physics models of such plasmas.

While the experiments discussed in $\S 2$ were performed with a gaseous-density target, the results described in this section were obtained by focusing a laser onto rotating solid-disk targets composed of various elements. In all of the following experiments, copious X-ray emission is characterized by use of temporally and spectrally resolved diagnostics.

In a related experiment, we followed the change in the observed series limits that occurs as carbon is heated and then decompresses over a range of electron density $n_{e}$ and temperature $T_{e}$ extending from $10^{23}$ to $10^{21} \mathrm{~cm}^{-3}$ and from 80 to $50 \mathrm{eV}$, respectively, which corresponds to $1.5>$ $\Gamma>0.5$. Making use of independent plasma diagnostics over this large range of parameter space, we demonstrate (Nantel et al. 1998) the validity of the Inglis-Teller limit for line-merging predictions.

In this experiment, a higher repetition rate $(10 \mathrm{~Hz}), 100 \mathrm{fs}$ Ti:sapphire laser was used. Pulses of $50 \mathrm{~mJ}$ in energy at 780 $\mathrm{nm}$ were focused with a $\mathrm{MgF}_{2}$ lens to an intensity of $10^{17} \mathrm{~W}$ $\mathrm{cm}^{-2}$. The use of subpicosecond pulses has the advantage of depositing the energy impulsively, in a thin target layer. Thus, the measurements are conducted after the laser pulse, in a freely decompressing ionized material. The emission spectra from the target were recorded with a grazingincidence flat-field-imaging XUV spectrometer coupled to an X-ray streak camera with CCD readout, for a spatial resolution of $80 \mu \mathrm{m}$ in the direction normal to the target plane and a spectral resolution of $0.15 \AA$. The subpicosecond X-ray streak camera was coupled to a jitter-free averaging sweep system (Maksimchuk et al. 1996), consisting of two photoconductive switches triggered by part of the laser beam to sweep the plates of the camera at $10 \mathrm{~Hz}$, in synchronization with the target emission. This novel technique - used for the first time here for spectroscopysignificantly extends the dynamic range of the streak camera and increases the signal-to-noise ratio. We were able to average the XUV spectrum over 600 shots with a temporal resolution of $4 \mathrm{ps}$, limited by a residual jitter of \pm 2 ps. The laser contrast was improved by a factor of more than 100 over the typical figure of $10^{5}$ for Ti:sapphire lasers through high-energy seeding of clean pulses in the regenerative amplifier. This new technique delivers a laser contrast of greater than $5 \times 10^{7}$ in order to allow the deposition of the laser energy at solid-target densities without the energy losses incurred by frequency doubling.

We present data from the $80 \mu \mathrm{m}$ region closest to the target plane. Figure 3 shows three spectra between 25 and $37 \AA$ taken at 0,50 , and 225 ps. These line-outs are integrated over $4 \mathrm{ps}$, and $t=0 \mathrm{ps}$ is arbitrarily defined as the time of the start of the emission. The principal emission lines of the H-like and He-like series are identified, as well as the $n=2 \mathrm{He}$-like satellites to the $\mathrm{H}$-like $2 p-1 s$ line. There is considerable free-bound continuum radiation ending at the He-like series limit near the $\mathrm{H}$-like $2 p-1 s$ line. The vacuum ionization potential (IP) of the He-like ion is marked at $31.62 \AA(392.09 \mathrm{eV})$, and the He-like radiative recombination continuum and edge are sketched in thick solid lines. The position of the detectable series limit (marked by vertical dotted lines for each spectrum) is determined to be $\approx 0.4-0.7 \AA$, limited by the presence of the unmerged bound-bound emission lines masking its exact position.

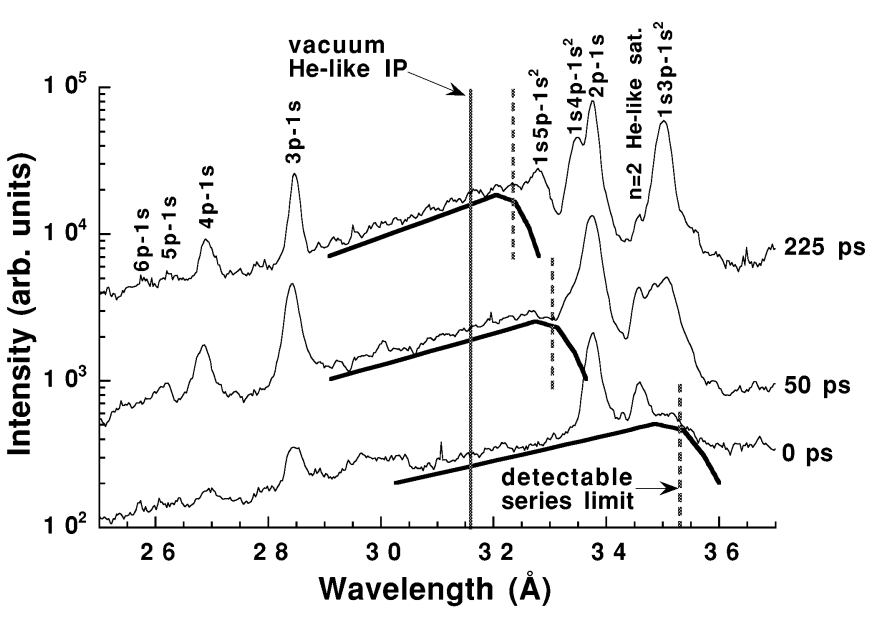

FIG. 3.-Line-outs from the time-resolved spectra taken at $t=0,50$, and 225 ps. The He-like $n p 1 s-1 s^{2}(n>3)$ and H-like $n p-1 s$ line emissions are identified as well as the $n=2 \mathrm{He}$-like satellites to the H-like $2 p-1 s$ line. The free-bound continuum and the detectable series limit are drawn on each line-out. Considerable He-like $2 p 1 s-1 s^{2}$ emission at $40.268 \AA$ was seen in each spectrum but is not included here, in order to emphasis better the spectral region between 25 and $37 \AA$. The three line-outs are offset vertically for better viewing.

This detectable He-like series limit is measured to move from $35.2 \AA(352.5 \mathrm{eV})$ at 0 ps to $33.1 \AA(374.3 \mathrm{eV})$ at $50 \mathrm{ps}$ and to $32.6 \AA(380.9 \mathrm{eV})$ at $225 \mathrm{ps}$. The separation between the vacuum IP and the observed edge for each spectrum indicates the combined effect on the spectrum of pressure ionization and line merging, which can move the observable series limit by as much as $40 \mathrm{eV}$ or past the He-like $3 p-1 s$ resonance line. No unmerged resonance lines from the He-like series are detected at 0 ps save the strong $2 p-1 s$ transitions (not shown in Fig. 3 for better viewing of the wavelength range near the series limit), and all the readily identifiable satellites to the $\mathrm{H}$-like $2 p-1 s$ line originate from $n=2$ doubly excited states. As the plasma evolves with time, the density - and hence the plasma field effectsgradually decreases and the ionic potential is less and less perturbed: identifiable line emissions from higher $n$ states reappear gradually, as is observed at 50 ps (an He-like $1 s 3 p$ $1 s^{2}$ line is clearly present, and an $1 s 4 p-1 s^{2}$ line is visible in the H-like $2 p-1 s$ low-wavelength shoulder) and at $225 \mathrm{ps}$ (He-like $1 s 4 p-1 s^{2}$ and $1 s 5 p-1 s^{2}$ lines are now clearly identifiable). A similar trend is observed in the higher $n$ $\mathrm{H}$-like emission lines, with the strengthening of the $3 p-1 s$ and $4 p-1 s$ lines and the appearance of the $5 p-1 s$ and $6 p-1 s$ lines in the later line-outs, despite the falling temperature evident from the steepening of the continuum slope.

In our analysis of the last experiment in $\S 3$, electron temperature and density diagnostics were obtained from the experimental spectra. We first established the electron temperature by the slope of the He-like continuum. We then generated artificial spectra using steady state non-LTE FLY simulations (Lee \& Larsen 1996) and found matches for the experimental spectra by varying the density in the simulation. This was done for eight line-outs at times 0,14 , $25,50,75,125,175$, and 225 ps after the start of the emission. The artificial spectra include instrumental broadening, pressure ionization, Stark broadening, and opacity. The different models of pressure ionization that were used for the generation of the excited-state populations and artificial spectra were the Stewart-Pyatt model (already included in 
FLY) and the ion-cell and Debye-Huckel models (implemented in FLY for this occasion); the Stark broadening took care of the line merging naturally. The positions of the He-like satellites to the $\mathrm{H}$-like $2 p-1 s$ line were defined originally in FLY for carbon through interpolation from similar lines in other elements, and they appeared at wavelengths about $0.35 \AA$ lower than in the experimental spectra or the Kelly tables. We corrected this in the code in order to permit a better comparison with the experimental data. Figure 4 gives an example of a match for the line-out at $t=225 \mathrm{ps}$ and a comparison of artificial spectra obtained with the different pressure ionization models. While the ioncell (IC, dashed curve) and Stewart-Pyatt (SP; solid curve) models give almost undistinguishable fits for similar densities, we found it impossible to match properly the artificial spectra generated with Debye-Huckel (DH) pressure ionization (dotted curve), regardless of the choice of density. This should not come as much of a surprise since the DH model should only be valid in the case of low-density plasmas.

Not only can these laser-produced X-rays be used in emission spectroscopy, they can also be used for absorption spectroscopy. In this way, recombination edge shifts, again characteristic of continuum lowering (pressure ionization), are observed at high plasma density and during shock formation (Workman et al. 1997).

Besides the Coulomb fields of unscreened adjacent ions, high fields can also be generated by strong electromagnetic radiation. Like the former, these latter fields can also perturb the energy-level structure of ions. As an example, we have shown that X-ray lines that are normally forbidden (by parity rules) are observed to appear, but only during the

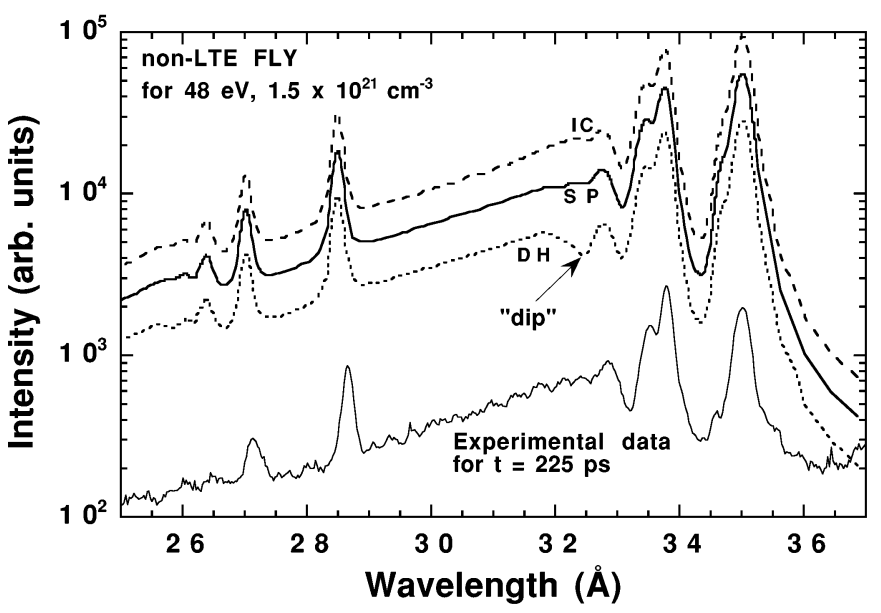

FIG. 4. - Comparison of a line-out at $t=225$ ps with non-LTE FLY simulations at $48 \mathrm{eV}$ (determined from the slope of the continuum) and $1.5 \times 10^{21} \mathrm{~cm}^{-3}$. Three different artificial spectra are shown, corresponding to the cases in which the continuum lowering is calculated with the IC (dashed curve), SP (solid curve), and DH (dotted curve) models. Note the conspicuous "dip" in the DH spectra near the He-like series limit. The line-outs are offset vertically for better viewing.

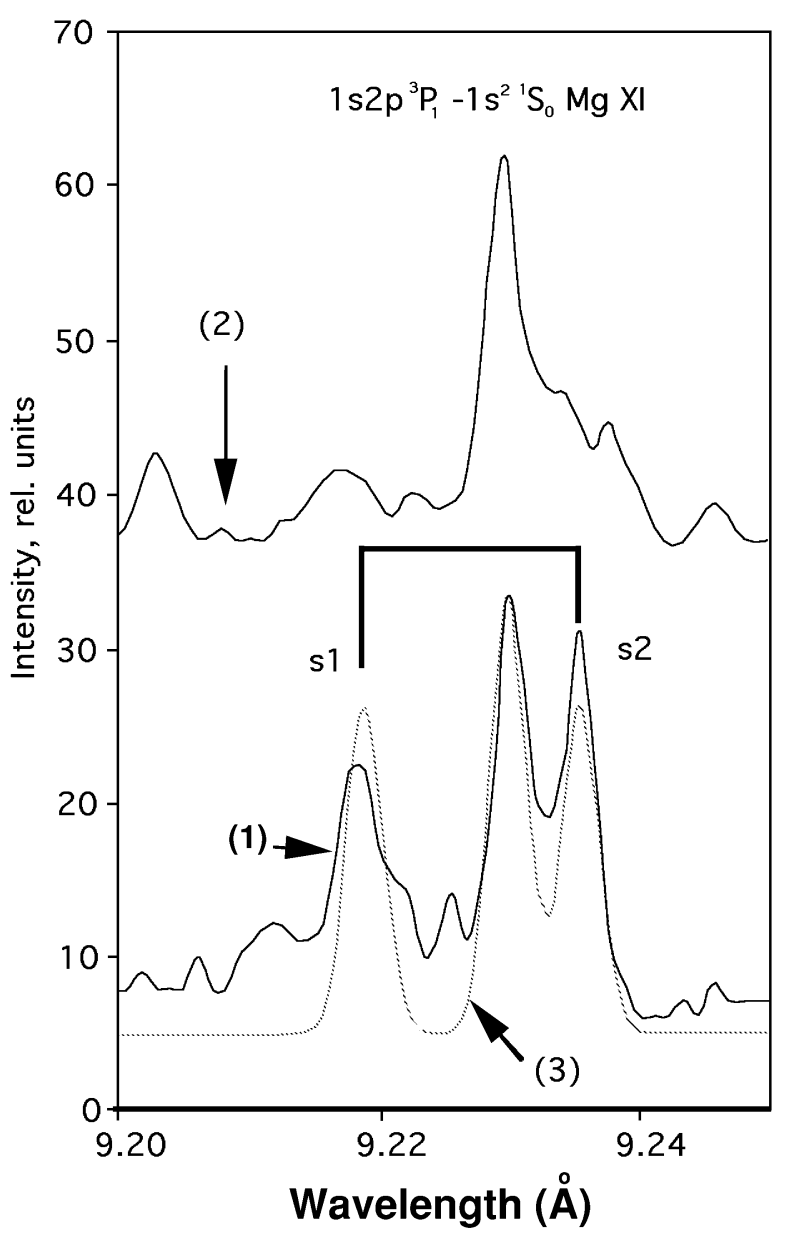

FIG. 5.-Radiation spectra from a magnesium plasma, during the laser (curve 1) and immediately after the pulse (curve 2). Curve 3 is the result of theoretical modeling. Note that the satellites are present only during the pulse.

laser pulse, because of the effects of the large laser electromagnetic field (100 $\mathrm{TeV} \mathrm{m}^{-1}$; Pikuz et al. 1997), as shown in Figure 5.

These Raman satellites are the result of dressing the ion Coulomb field with the electric field of the laser. The observation of such satellites may someday prove to be a useful diagnostic of the presence of strong fields in astrophysical bodies.

For the work on dense plasmas, we gratefully acknowledge the support of the Division of Chemical Sciences, Office of Basic Energy Sciences, Office of Science, US Department of Energy. For the work on relativistic plasmas and the laser facilities, we gratefully acknowledge the support of the National Science Foundation.

\section{REFERENCES}

Barnes, D. C., Kurki-Suonio, \& Tajima, T. T. 1987, IEEE Trans. Plasma Sci., PS-15, 154

Chen, S.-Y., Sarkisov, G. S., Maksimchuk, A., Wagner, R., \& Umstadter, D. 1998, Phys. Rev. Lett., 80, 2610

DaSilva, L., et al. 1989, Phys. Rev. Lett., 62, 1623
Ichimaru, S. 1982, Rev. Mod. Phys., 54, 1017

Leboucher-Dalimier, E., et al. 1994, J. Quant. Spectrosc. Radiat. Transfer, 51,187

Lee, R. W., \& Larsen, J. T. 1996, J. Quant. Spectrosc. Radiat. Transfer, 56, 535 
Maksimchuk, A., et al. 1996, Rev. Sci. Instrum., 67, 697

Nantel, M., Ma, G., Gu, S., Cote, C. Y., Itatani, J., \& Umstadter, D. 1998, Phys. Rev. Lett., 80, 4442

Pikuz, S. A., Maksimchuk, A., Umstadter, D., Nantel, M., Skobelev, I. Yu.,

Faenov, A. Ya., \& Osterheld, A. 1997, Pisma v ZhETP, 66, 454

Pukhov, A., \& Meyer-ter-Vehn, J. 1996, Phys. Rev. Lett., 76, 3975

Umstadter, D., Chen, S.-Y., Maksimchuk, A., Mourou, G., \& Wagner, R.

1996, Science, 273, 472
Umstadter, D., \& Norris, T., eds. 1997, IEEE J. Quantum Electron., 33, 1878-1968

Wagner, R., Chen, S.-Y., Maksimchuk, A., \& Umstadter, D. 1997, Phys. Rev. Lett., 78, 3125

Workman, J., Nantel, M., Maksimchuk, A., \& Umstadter, D. 1997, Appl. Phys. Lett., 70, 312 